\title{
Activités
}

4-1 | avril 2007

ATWAD

\section{Analyser l'activité de médecins hospitaliers pour concevoir la formation : le cas de la prescription de neuroleptiques}

Analysis of medical practices for building physicians' education: the prescription

of antipsychotic drugs

Analizar la actividad de los médicos de hospitales para poder diseñar la

capacitación: el caso de la prescripción de neurolépticos

\section{Aurélie Grass, Michel Grangeat et Benoît Allenet}

\section{OpenEdition}

\section{Journals}

Édition électronique

URL : http://journals.openedition.org/activites/1380

DOI : 10.4000/activites. 1380

ISSN : 1765-2723

Éditeur

ARPACT - Association Recherches et Pratiques sur les ACTivités

\section{Référence électronique}

Aurélie Grass, Michel Grangeat et Benoît Allenet, «Analyser l'activité de médecins hospitaliers pour concevoir la formation : le cas de la prescription de neuroleptiques ", Activités [En ligne], 4-1 | avril 2007, mis en ligne le 15 avril 2007, consulté le 30 avril 2019. URL : http://journals.openedition.org/ activites/1380 ; DOI : 10.4000/activites. 1380

\section{c) (i) $(9)$}

Activités est mis à disposition selon les termes de la licence Creative Commons Attribution - Pas d'Utilisation Commerciale - Pas de Modification 4.0 International. 


\title{
Analyser l'activité de médecins hospitaliers pour concevoir la formation : le cas de la prescription de neuroleptiques
}

\author{
Aurélie Grass \\ Docteur en Pharmacie, Pharmacie du centre hospitalier, 38120 Saint-Égrève. \\ grass.aurelie@wanadoo.fr \\ Michel Grangeat \\ Maître de conférences, Laboratoire des Sciences de l'Éducation (LSE - EA 602), Université Pierre Mendès France et \\ IUFM. 38000 Grenoble. \\ michel.grangeat@upmf-grenoble.fr \\ Benoît Allenet \\ Maître de conférences, ThEMAS TIMC-IMAG (UMR CNRS 5525), Université Joseph Fourrier, Département de \\ pharmacie, Centre hospitalier universitaire. 38000 Grenoble. \\ BAllenet@chu-grenoble.fr
}

\begin{abstract}
Analysis of medical practices for building physicians' education: the prescription of antipsychotic drugs. The present study aims at understanding the observed gap between routine medical practice of some French physicians working in a psychiatric hospital and the available clinical guidelines Based on the theoretical field of didactic including conceptualisation, semi directed interviews were conducted with 12 physicians working in the same psychiatric hospital, chosen depending on their seniority and expertise levels. The results allow building the referential for their clinical activity and outlining what is needed regarding the training of these physicians.
\end{abstract}

KEYWORDS

didactics, skills, conceptualisation, schizophrenia, semi directed interviews, medical prescription of antipsychotic drugs.

En médecine, conférences de consensus et recommandations pour la pratique clinique synthétisent les recommandations consensuelles émises par les sociétés savantes médicales, avec le soutien de la Haute Autorité en Santé (HAS) afin de fournir des repères objectifs et indépendants aux médecins. Réactualisés régulièrement, ces textes sont largement diffusés. Néanmoins, des études montrent que les pratiques médicales s'écartent des guides de bonnes pratiques, dans différentes disciplines telles que la médecine d'urgence, la chirurgie, la cardiologie (Staikowski, Chouaid, Zanker, Pevirieri, Traineau, \& Lagha, 1997; Demange, Di Sandro, Coince, \& Philippe, 1999; Bedouch, Labarère, Chripaz, Allenet, Lepape, Fourny et al., 2004). C'est également le cas pour la psychiatrie, concernant la prise en charge de la schizophrénie (Glikman, Pazart, Casadebaig, Philippe, Lachaux, Kovess et al. 1999). Pourtant, l'évaluation des pratiques médicales visant l'amélioration de la qualité des soins par l'harmonisation de pratiques - jusque-là hétérogènes entre elles et par rapport aux données de la science - est devenue depuis peu obligatoire pour tout médecin (loi du 13 août 2004). Elle est d'ailleurs partie intégrante de la procédure d'accréditation à laquelle chaque établissement de santé est périodiquement soumis (HAS, 2005).

L'objet de la présente étude est de comprendre les processus qui déterminent ces écarts. 


\section{1.- Le contexte des pratiques de prescription de neuroleptiques}

Le cas de la schizophrénie - une pathologie centrale dans notre étude - s'inscrit dans ce problème général. Associant délire, hallucinations, désorganisation du discours et des comportements, cette maladie chronique évolue vers une dissociation de la personnalité avec déficit mental (Godfryd, 1993). Sa prise en charge psychiatrique a pour but de conduire le patient, sinon à la guérison, tout au moins à un état d'équilibre permettant sa sortie de l'hôpital (Chabannes, Benattia, Pascal, Azorin, Vidon, Bouhassira et al., 1998). Associés aux régulateurs de l'humeur, antidépresseurs et anxiolytiques, les neuroleptiques représentent l'essentiel du traitement (Bourgeois, 2003). Leurs effets indésirables potentiels nécessitent une surveillance spécifique aux niveaux pondéral, cardiaque, sanguin, neurologique et clinique (Reynaud, 1994 et Gury, Ames, Camm, Cook, Falkai, Hurley et al., 2003). Néanmoins, une récente étude (Ménard, \& Roy, 2004) montre, après l'étude de Glikman et al. (1999), que les pratiques de prescription et de suivi des médicaments neuroleptiques s'écartent là encore des recommandations ; or, ces dernières sont formalisées par une conférence de consensus réactualisée l'année même de cette enquête (Fédération Française de Psychiatrie, 2003).

L'activité du médecin psychiatre doit toutefois être resituée dans son contexte organisationnel. Le récent plan de santé mentale 2005-2008, mis en place pour «donner à la psychiatrie française un nouveau souffle » intervient alors que, depuis une vingtaine d'années, le recours à la psychiatrie est en constante augmentation, que la réduction régulière et continue des places en hôpital n'a pu être que partiellement compensée par les réseaux de prise en charge extra hospitaliers et que l'évolution des populations médicale et infirmière laisse présager une baisse problématique pour les années à venir (Ministère français des solidarités, de la santé et de la famille, 2005).

L'écart entre les pratiques attendues et réelles qui est étudié dans ce texte s'inscrit donc, à la fois, dans une activité complexe - la prescription de neuroleptiques - et dans un contexte organisationnel difficile - la réorganisation des moyens.

\section{1.- Cadre conceptuel de l'étude : activité constructive et situation de travail}

L'activité médicale est vue ici comme toute activité professionnelle. En conséquence, nous proposons de l'étudier selon le cadre de la didactique professionnelle et, plus largement, de celui de la psychologie ergonomique qui en est en partie à l'origine (Rogalski, 2004). Deux concepts principaux seront mobilisés : celui d'activité et celui de situation.

Comme pour d'autres activités professionnelles, on considère que l'activité des médecins s'inscrit dans la tâche prescrite tout en la dépassant (Leplat, 1997) : elle déborde la tâche et son exécution par l'agent en prenant en compte ses représentations mentales, ses modèles cognitifs et ses motivations. Elle se décompose en activité productive, visant à l'obtention de résultats, et en activité constructive, perdurant au-delà de l'action, soutenant le développement des compétences. Cette dernière - issue de l'expérience personnelle, durant les études, la vie sociale et le métier - permet à l'agent de faire face aux diverses situations de travail auxquelles il est confronté (Samurçay, \& Rabardel, 2004).

Cet aspect cognitif, constructif, de l'activité est décrit dans la littérature comme étant sous-tendu par des processus de conceptualisation qui permettent à l'agent de faire le lien entre ses connaissances et ses actions afin de mieux contrôler ses démarches, d'adapter ses manières de faire aux particularités de la situation (Vergnaud, 1996). Les schèmes - éléments cognitifs élaborés par l'agent en situation - représentent le support de cette conceptualisation. Ils permettent, notamment, à l'agent de redéfinir la tâche prescrite, en partie implicite puisque rarement décrite en totalité. Ils sont décrits comme étant constitués de quatre éléments complémentaires : les buts qui orientent l'action, les indices ou événements tirés de la situation qui génèrent cette action, les règles d'action qui en assurent la réalisation et les savoirs qui justifient les choix parmi l'ensemble des règles d'action potentiellement adéquates. Ces éléments cognitifs, ces savoirs d'action (Barbier, \& Galatanu, 2004), s'ils demeurent isolés n'autorisent qu'une conduite de l'activité au coup par coup. De fait, avec l'expérience et la 
formation, ils sont organisés et hiérarchisés en constituant des modèles opératifs, des répertoires permettant à l'agent de planifier son activité en s'adaptant aux situations rencontrées, même nouvelles. Le modèle opératif permet ainsi de catégoriser les actions autour des dimensions essentielles de l'activité professionnelle (Pastré, 2004, 2005). Il permet aussi de donner du sens à ces actions en les intégrant à des entités plus larges, telles que la connaissance de la situation de travail, la prise en compte des longues temporalités régissant les actions en cours ou les savoirs sur le métier. Le niveau de compétence de l'agent est alors fonction de l'organisation de ce modèle opératif : celui du novice est centré sur des détails, sans vraiment de liaisons en entre eux ; celui de l'expert est à la fois étendu et hiérarchisé. Cependant, tous les modèles opératifs de tous les agents ne peuvent se construire indépendamment les uns des autres, de la tâche prescrite ou des savoirs de métier. On définit alors un modèle commun - nommé structure conceptuelle de la situation - que l'on décrit à partir du point de vue des experts.

La situation détermine cependant le développement des compétences et donc des processus de conceptualisation. Dans le cas de cette étude de praticiens en institution, l'activité se place dans un contexte collectif et la nature de l'évolution du professionnel au sein d'un réseau de collègues détermine les compétences (Zarifian, 2004). Deux positionnements dans le collectif sont retenus ici, selon que le sujet se limite au noyau dur du métier, au cœur de la tâche prescrite, ou que, à l'inverse, il profite des interactions avec d'autres collègues ou d'autres services pour améliorer l'efficacité de son action (Leplat, 1997). Ce collectif fonctionne à partir d'une représentation opérative partagée qui permet aux individus de coordonner leurs actions (Rogalski, 2005) : plus cette représentation - que l'on assimilera ici à la structure conceptuelle de la situation - est largement partagée, plus l'activité collective est efficace.

La pratique étudiée ici se situe également en contexte dynamique (Amalberti, 2001). Quatre caractéristiques de ce type de contexte seront retenues : les actions requises des agents ne peuvent être décrites en détail car elles dépendent d'une multitude de facteurs contextuels; les indices à partir desquels l'agent peut réguler son action ne représentent que des indicateurs pour des variables sur lesquelles nul ne peut jouer directement; le système évolue de lui-même sans action de l'agent, voire en contrecoup de cette action; la conduite efficace est celle qui consiste à anticiper l'apparition de dysfonctionnements, en maintenant le système dans un état d'équilibre acceptable ne générant pas de risques, quitte à ne pas respecter strictement la procédure prédéfinie (Hoc, Amalberti, Cellier, \& Grosjean, 2004).

Une telle étude des compétences, dans le cadre de la didactique professionnelle, ne vise donc pas à évaluer le sujet, à le situer sur une échelle entre novice et expert par exemple. Elle vise à comprendre les processus qui déterminent l'activité afin de transformer les situations de travail et de concevoir des formations en adéquation avec les prescriptions, certes, mais aussi avec les conditions locales de leur mise en œuvre.

Nous tenterons ici d'utiliser ces concepts, qui ont fait leurs preuves dans de nombreuses professions, afin d'analyser l'activité médicale. Plusieurs raisons laissent penser que cette approche pourrait s'avérer pertinente. Tout d'abord, bien que la profession soit encadrée par de nombreuses références juridiques et recommandations, sous la forme de conférences de consensus et de référentiels locaux, les opérations à réaliser par le médecin sont rarement décrites en détail. De fait, la prescription médicamenteuse, prérogative médicale, est souvent décrite comme une pratique idéalement basée sur les preuves apportées par la science, mais dans laquelle le professionnel se doit d'appliquer la meilleure preuve disponible pour traiter son patient (Sackett, Rosenberg, Gray, Haynes, \& Richardson, 1996 ; Lachaux, Pasi-Delay, \& Adouard, 1998). En outre, le médecin est un organisateur, articulant les interventions de différents professionnels de santé et équipes de soins pour la prise en charge optimale du patient (Pasi-Delay, \& Lachaux, 1998 ; Gury, Ames, Camm, Cook, Falkai, Hurley et al., 2003). L'étendue des situations cliniques possibles introduit ainsi un haut degré de complexité : les démarches à mettre en œuvre ne pouvant être toutes envisagées et décrites, le professionnel est confronté à des situations nouvelles caractérisées par leur grande diversité et leur écart aux situations basiques du 
métier. Enfin, l'évolution du patient entre deux consultations médicales, indépendamment de l'action du professionnel, confère un aspect dynamique à l'activité médicale (Marquié, Raufaste, Mariné, \& Ecoiffier, 2003).

En conséquence, la pratique médicale peut être analysée comme une activité collective - dont l'étude est bien documentée dans de nombreuses professions (Rogalski, 1995 ; Hatchuel, 1996 ; Weill-Fassina, \& Benchekroun, 2000 ; Grangeat, \& Chakroun, 2005) - et comme une activité en situation dynamique - de la même manière que l'enseignement (Rogalski, 2003 ; Grangeat, 2006) ou la conduite de systèmes technologiques de pointe (Samurçay, \& Hoc, 1988 ; Pastré, 1999a, 1999b; Hoc, et al. 2004). De fait, certaines recherches dans le domaine médical s'inspirent de la didactique professionnelle ou de la psychologie ergonomique (Faure, \& Gonzalez, 1988 ; Boreham, Foster, \& Mawer, 1992 ; Boreham, Mawer, \& Foster, 1996 ; Anceaux, \& Beuscart-Zéphir, 2002 ; Boreham, 2002 ; Beaulieu, Rivard, Hudon, Saucier, Remondin, \& Favreau, 2003 ; Spies, Mokkink, de Vries Robe, \& Grol, 2004).

\section{2.- Problématique et méthodologie}

Face au constat d'écart entre les pratiques observées et les recommandations institutionnelles, nous visons à analyser l'activité médicale afin de mettre en évidence les compétences de médecins dans le suivi de patients sous neuroleptiques et d'esquisser des organisations de travail propices à leur développement.

Cette étude nécessite l'élaboration d'un référentiel préalable, à partir d'informations sur la tâche prescrite, recueillies lors d'une revue de la littérature médicale. Les déterminants de l'activité apparaissent, effectivement, comme étant très divers : influences respectives de la formation initiale et continue du médecin (Lachaux et al. 1998 ; Ray-Coquard, Chauvin, Lurkin, Ducimetière, Jacquin, Agostini et al., 2006 ; code de déontologie médicale), de l'expérience acquise lors de l'exercice professionnel (Prosser, \& Walley, 2006), du côtoiement des visiteurs médicaux réalisant l'essentiel de la publicité autour du médicament (La Revue Prescrire, 2006), des collègues exerçant dans l'institution, dans les hôpitaux voisins, en libéral (Pasi-Delay, \& Lachaux, 1998 ; Sauvagnac, Beckendorf, Lesur, Luporsi, Stines, Falzon, et al., 1999; Gury et al., 2003), des conditions matérielles locales lors de l'exercice de l'activité (Ray-Coquard et al., 2006), des exigences législatives et réglementaires encadrant la profession. Un référentiel des déterminants de l'activité peut cependant être structuré selon trois axes : l'expérience professionnelle, les références législatives et réglementaires propres à la profession et les conditions matérielles de travail. Ces axes définissent trois thèmes principaux qui se déclinent en douze sous-thèmes à aborder lors de l'entretien avec le médecin ; l'ensemble structure l'investigation (cf. Annexe 1).

\section{1.- Appui des entretiens sur une trace de l'activité}

Les recherches dans le domaine médical qui se réfèrent au cadre théorique proposé ici utilisent divers modes d'accès à l'activité : étude de dossiers médicaux, auto-questionnaire, entretien d'autoconfrontation, participation de patients standards, utilisation de cas cliniques factices, utilisation de la technique de verbalisation simultanée. Dans le cas de la présente étude, l'enregistrement ou l'observation de médecins, durant leur activité en psychiatrie, ne sont pas envisageables. De ce fait, le recueil des données s'effectue par entretiens semi-structurés dits d'explicitation, conçus pour obtenir la verbalisation a posteriori du vécu de l'action et, ainsi, accéder aux structures cognitives mises en jeu (Vermersch, 2004).

Lors de l'entretien, afin de confronter le médecin à une trace de son activité, le recours à une simulation inspirée d'un cas réel a été envisagé. Faisant partie des épreuves du concours d'internat en médecine, le commentaire de cas cliniques est familier au médecin. Cependant, le professionnel confronté 
à une ordonnance factice pourrait être incité à exposer des savoirs théoriques éloignés de son activité. C'est pourquoi nous avons choisi de confronter chaque médecin à une ordonnance qu'il avait rédigée lui-même pour l'un de ses patients. Utiliser de telles ordonnances comme supports d'entretien, jouant un rôle d'amorce et de lien entre la situation professionnelle antérieure et sa verbalisation, devrait permettre d'accéder à une part de l'activité tout en évitant les propos trop convenus. Cependant, afin d'éviter de donner l'impression de prendre le sujet en défaut et, ainsi, de provoquer des jugements sur l'activité et des rationalisations a posteriori, les ordonnances choisies sont conformes à ce qui peut être attendu par l'institution de l'interviewé.

Le guide d'entretien, qui s'appuie sur le référentiel, comporte l'énoncé d'un contrat de communication préalable à l'entretien, rappelant notamment l'objet de la recherche et le déroulement de l'entretien, suivi de douze questions ou relances, permettant d'aborder les douze sous-thèmes retenus. Le protocole de recherche, utilisant une ordonnance réelle et le guide d'entretien, a été validé lors d'un entretien préliminaire avec un médecin psychiatre d'une institution psychiatrique voisine.

Ce protocole de recherche respecte différentes exigences éthiques : les médecins rencontrés sont tous volontaires pour recevoir le chercheur; ils acceptent l'enregistrement des échanges avec celui-ci. Par ailleurs, les données relatives au patient sont soumises au secret professionnel que le chercheur s'engage à respecter.

Le nombre de médecins inclus dans l'étude est de douze, exerçant dans une même institution psychiatrique, choisis selon leur ancienneté dans la profession - juniors (i.e : internes, encore étudiants) / seniors (i.e : praticiens hospitaliers, médecins diplômés) - et leur spécialité médicale - psychiatres / « somaticiens » (i.e : médecins, généralistes, neurologues, autres que psychiatres).

\section{2.- Identification de savoirs d'action}

Les entretiens sont enregistrés pour être retranscrits en vue d'une analyse de contenu, permettant de caractériser l'activité de suivi des patients sous neuroleptiques telle qu'elle est pensée et dite par le médecin. Le discours est segmenté en épisodes correspondant aux indicateurs du référentiel préalable. L'expression de la conceptualisation est identifiée sous la forme de savoirs d'action : pour chacun des épisodes, les éléments du discours faisant référence aux quatre constituants du schème sont recherchés (Grangeat, \& Munoz, 2006). Un savoir d'action est défini comme « incomplet» lorsque tous les constituants du schème ne sont pas identifiés dans le discours et comme « hiérarchisé » lorsque plusieurs règles d'action potentielles correspondent à un but commun. Les savoirs d'action ont été classés en catégories correspondant chacune à une règle d'action énoncée.

\section{3.- Identification des experts}

Les savoirs d'action des experts permettront d'appréhender la structure conceptuelle de la situation étudiée. En s'appuyant sur les pratiques des médecins les plus compétents et par l'analyse des différences observées entre les experts et les autres professionnels, le chercheur établit un référentiel de l'activité qui pourra servir de base à des formations ultérieures (Coulet, \& Chauvigné, 2005). Cependant, comme dans tout métier de relations humaines, il est difficile de repérer des experts sans être perturbé par des biais institutionnels (grade, ancienneté).

Ici, les experts ont été identifiés grâce à une combinaison de quatre indicateurs spécifiquement élaborés pour la recherche. Premièrement, l'implication du professionnel dans son activité : elle est déterminée au regard du cursus universitaire puis professionnel, de la régularité de la formation personnelle, de la participation à des structures professionnelles ou à des activités d'enseignement ou de recherche. Un score est attribué à chaque sujet, selon une grille de cotation spécifique (cf. Annexes 2 et 3). Deuxièmement, le nombre de savoirs d'action identifiés dans la transcription de l'entretien avec le professionnel. Troisièmement, la proportion de savoirs d'action hiérarchisés. Quatrièmement, le positionnement équilibré du professionnel par rapport aux trois dimensions de l'activité. Pour 
chaque indicateur, un seuil est fixé pour chacune des trois variables, en fonction de la médiane de la variable considérée (cf. Tableau 2).

\section{3.- Résultats}

Douze entretiens de 20 minutes en moyenne ont été menés auprès de trois internes en psychiatrie, trois internes de médecine générale, trois psychiatres praticiens hospitaliers seniors et trois médecins « somaticiens » seniors.

\section{1- Données recueillies lors des entretiens}

Dans le corpus des entretiens, 921 épisodes formant 174 savoirs d'action ont été identifiés. Dans les exemples ci-après (cf. Tableau 1), le $\mathrm{n}^{\circ} 1$ est un savoir d'action ordinaire, le $\mathrm{n}^{\circ} 2$ est hiérarchisé et le $\mathrm{n}^{\circ} 3$ incomplet.

\begin{tabular}{|c|c|c|c|c|}
\hline Exemples & $\begin{array}{c}\text { Indices pris dans la } \\
\text { situation } \\
\end{array}$ & But & Règle d'action & Justification \\
\hline$n^{\circ} 1$ & $\begin{array}{l}\text { Chez les gens sans } \\
\text { facteurs de risque } \\
\text { cardiovasculaire, qui } \\
\text { sont sportifs, jeunes }\end{array}$ & $\begin{array}{l}\text { pour ne pas être } \\
\text { trop systématique }\end{array}$ & $\begin{array}{l}\text { il y a des éléments } \\
\text { qu'on prend en } \\
\text { compte }\end{array}$ & $\begin{array}{l}\text { ma politique au niveau des } \\
\text { examens paracliniques, } \\
\text { qu'ils soient biologiques } \\
\text { ou autres, c'est plutôt que } \\
\text { ce ne soit pas systématique, } \\
\text { bête et méchant }\end{array}$ \\
\hline \multirow[b]{3}{*}{$n^{\circ} 2$} & \multirow{2}{*}{$\begin{array}{l}\text { dès qu'il y a besoin par } \\
\text { rapport à l'épilepsie }\end{array}$} & \multirow{3}{*}{$\begin{array}{l}\text { par rapport à } \\
\text { des avis plus } \\
\text { spécialisés }\end{array}$} & \multirow{2}{*}{$\begin{array}{l}\text { on fait beaucoup } \\
\text { appel à la } \\
\text { neurologue }\end{array}$} & $\begin{array}{l}\text { elle interprète les } \\
\text { électroencéphalogrammes }\end{array}$ \\
\hline & & & & on s'entend bien avec elle \\
\hline & donc à ce moment-là & & $\begin{array}{l}\text { on peut aussi } \\
\text { initier une } \\
\text { consultation } \\
\text { spécialisée, surtout } \\
\text { avec la neurologue }\end{array}$ & $\begin{array}{l}\text { la majorité des effets } \\
\text { secondaires des } \\
\text { neuroleptiques qu'on } \\
\text { observe sont neurologiques }\end{array}$ \\
\hline \multirow{2}{*}{$n^{\circ} 3$} & \multirow{2}{*}{ quand il y a besoin } & $\begin{array}{l}\text { pour un problème } \\
\text { que je ne sais pas } \\
\text { régler }\end{array}$ & \multirow{2}{*}{$\begin{array}{l}\text { on fait appel aux } \\
\text { somaticiens }\end{array}$} & \multirow{2}{*}{$\begin{array}{l}\text { non identifiée dans le } \\
\text { discours }\end{array}$} \\
\hline & & $\begin{array}{l}\text { ou qui concerne } \\
\text { le somatique } \\
\text { purement }\end{array}$ & & \\
\hline
\end{tabular}

Tableau 1 : Trois types de savoir d'action

\section{2- Caractéristiques générales de l'activité}

Chacun des savoirs d'action identifiés a été catégorisé, selon sa règle d'action et son but. Tous les savoirs d'action ont pu être rangés dans l'une des trois catégories suivantes, données par ordre décroissant d'importance dans les entretiens : la surveillance du patient ( 75 savoirs d'action référencés, 43 $\%$ ), l'interaction avec d'autres médecins pour l'organiser (52 occurrences, $30 \%$ ) et enfin la collecte d'informations relatives à cette activité (47 occurrences, $27 \%$ ). Chacune des dimensions se compose de différentes actions, se divisant elles-mêmes en autant de règles d'action différentes exprimées par les professionnels durant les entretiens. 
La dimension 1, «Surveillance du patient », comprend quatre actions : adapter la nature et la fréquence du suivi aux signes biologiques et/ou cliniques, faire un bilan dès que possible, suivre l'efficacité et/ou la tolérance au long cours et continuer le suivi au long cours. La dimension 2, «Interactions avec d'autres médecins », inclut les quatre actions suivantes : déléguer, échanger, prendre conseil, coopérer. Enfin, la dimension 3, «Recherche d'informations, » se décline en six actions : lire, rencontrer des visiteurs médicaux, rencontrer des collègues, acquérir un esprit critique, acquérir une routine de fonctionnement grâce à son expérience, avoir différentes sources d'information.

Dans 66\% des cas, le patient représente le point de départ de l'action par le biais de :

ses signes cliniques ou biologiques («si le patient se plaint de tachycardie», " chez un patient sans antécédents cardiaques », "s'il y a des problèmes de santé indéfinis »),

son traitement (« les patients sous neuroleptiques », « pour tout médicament neuroleptique », « quand c'est une instauration de traitement »).

Viennent ensuite les conditions d'exercice propres à l'institution (« dans le recrutement que l'on a ici »). Enfin apparaît la notion d'urgence (« dans les situations de crise », « des patients en décompensation aiguë ») que les règles d'action correspondantes identifient à un facteur limitant l'action ( «l'électrocardiogramme n'est pas fait tout de suite, le premier n'est pas toujours fait, il est fait dans les jours qui suivent, dès que possible », " on a vraiment beaucoup de mal à les piquer »).

L'analyse des savoirs de référence montre la prépondérance du recours à l'expérience comme justification et explication de l'action ( $23 \%$ des références). Ce résultat laisse prévoir des difficultés pour les professionnels les plus jeunes, comme cette résidente en médecine à la veille de son diplôme qui déclare : "J'ai tellement de choses à apprendre, le moindre petit bout d'information est souvent le bienvenu. Et puis la pratique, ça ne se remplace pas!». Les autres savoirs de référence évoqués se partagent entre effets attendus du médicament, recommandations et textes juridiques disponibles.

\section{3.- Identification des experts}

La méthode utilisée fait apparaître trois catégories de professionnels (cf. Tableau 2). Les médecins répondant à plus de trois indicateurs sur quatre sont sélectionnés comme étant les experts (médecins $8,9,10,12)$. Deux autres catégories sont dégagées : les professionnels répondant à deux indicateurs sur quatre, dénommés professionnels expérimentés (médecins $5,6,7,11$ ) ; les professionnels répondant à un indicateur, voire à aucun, dénommés professionnels novices dans la profession (médecins $1,2,3,4)$.

\begin{tabular}{|c|c|c|c|c|}
\hline & \multicolumn{4}{|c|}{ Indicateur } \\
\hline Sujets & $\begin{array}{c}\mathrm{CV} \\
\text { score }>9\end{array}$ & $\begin{array}{c}\text { Nombre de savoirs } \\
\text { d'action }>17\end{array}$ & $\begin{array}{c}\text { Pourcentage de savoirs } \\
\text { d'action hiérarchisés }>60 \%\end{array}$ & $\begin{array}{l}\text { Positionnement homogène } \\
\text { au sein des trois dimensions }\end{array}$ \\
\hline Médecin 1 & & $\bar{X}$ & & \\
\hline Médecin 2 & & & & \\
\hline Médecin 3 & $\mathrm{X}$ & & & \\
\hline Médecin 4 & & & $\mathrm{X}$ & \\
\hline Médecin 5 & & & $\mathrm{X}$ & $\mathrm{X}$ \\
\hline Médecin 6 & $\mathrm{X}$ & $\mathrm{X}$ & & \\
\hline Médecin 7 & $\mathrm{X}$ & & $\mathrm{X}$ & \\
\hline Médecin 8 & $\mathrm{X}$ & $\mathrm{X}$ & $\mathrm{X}$ & $\mathrm{X}$ \\
\hline Médecin 9 & $\mathrm{X}$ & $\mathrm{X}$ & & $\mathrm{X}$ \\
\hline Médecin 10 & & $\mathrm{X}$ & $\mathrm{X}$ & $\mathrm{X}$ \\
\hline Médecin 11 & & $\mathrm{X}$ & $\mathrm{X}$ & \\
\hline Médecin 12 & $\mathrm{X}$ & $\mathrm{X}$ & $\mathrm{X}$ & $\mathrm{X}$ \\
\hline
\end{tabular}

Tableau 2 : Identification des trois catégories de professionnels 
Les experts sont deux psychiatres seniors, un interne en psychiatrie et une interne en médecine générale, ayant tous plus de deux ans d'ancienneté dans l'institution. Les novices sont deux somaticiens seniors, un interne en psychiatrie et un interne en médecine générale, qui ont tous moins de six mois d'ancienneté. Une catégorie intermédiaire - dite expérimentée - correspond à deux psychiatres (un interne, un praticien hospitalier) et à deux somaticiens seniors.

\section{4.- Trois manières de conduire l'activité}

Pour chacune des trois dimensions de l'action, l'analyse des répertoires de savoir-faire permet de comprendre comment les professionnels conduisent leur activité (cf. Tableau 3).

\begin{tabular}{|l|c|c|c|}
\hline & \multicolumn{3}{|c|}{$\begin{array}{c}\text { Nombre de professionnels concernés, } \\
\text { par catégorie d'expertise, pour chacun des critères }\end{array}$} \\
\hline \multicolumn{1}{|c|}{ Actions décrites au sein de chaque dimension } & $\begin{array}{c}\text { Novices } \\
(\mathrm{n}=4)\end{array}$ & $\begin{array}{c}\text { Expérimentés } \\
(\mathrm{n}=4)\end{array}$ & $\begin{array}{c}\text { Experts } \\
(\mathrm{n}=4)\end{array}$ \\
\hline \multicolumn{3}{|c|}{ Dimsion $\mathbf{~ : ~ S u i v i ~ d u ~ p a t i e n t ~}$} \\
\hline $\begin{array}{l}\text { Adapter la nature et la fréquence du suivi aux } \\
\text { signes biologiques et/ou cliniques }\end{array}$ & 3 & 2 & 4 \\
\hline Faire un bilan dès que possible & 3 & 2 & 4 \\
\hline Suivre l'efficacité et/ou la tolérance du traitement & 1 & 2 & 4 \\
\hline Continuer le suivi au long cours & 2 & 1 & 2 \\
\hline \multicolumn{1}{|c|}{ Dimension 2: Interaction avec un autre médecin } \\
\hline Déléguer & 4 & 3 & 1 \\
\hline Échanger & 1 & 3 & 3 \\
\hline Prendre conseil & 1 & 0 & 2 \\
\hline Coopérer & 0 & 1 & 3 \\
\hline & 2 & 4 & 2 \\
\hline Lire & 0 & 3 & 4 \\
\hline Rencontrer des visiteurs médicaux & 0 & 1 & 2 \\
\hline Rencontrer des collègues & 2 & 1 & 2 \\
\hline Acquérir un esprit critique & 0 & 1 & 3 \\
\hline $\begin{array}{l}\text { Acquérir une routine de fonctionnement grâce à } \\
\text { son expérience }\end{array}$ & 0 & 0 & \\
\hline Avoir différentes sources d'information & Recherche d'informations & \\
\hline
\end{tabular}

Tableau 3 : Comparaison des professionnels selon le degré d'expertise et pour chacune des trois dimensions de l'activité

Indication de lecture : Dans la Dimension 1, 3 novices sur 4 disent réaliser un bilan dès que possible contre 2 professionnels expérimentés sur 4 et 4 experts sur 4 .

Dans la dimension 1, « Suivi du patient », trois novices sur quatre disent réaliser un bilan dès que possible et l'adapter à l'évolution du patient. Un sur quatre dit évaluer l'efficacité ou la tolérance du traitement instauré et la moitié dit continuer le suivi au long cours. La moitié des médecins expérimentés, au plus, disent pratiquer des bilans et évaluer l'efficacité du traitement. En revanche, tous les experts disent faire réaliser un bilan adapté au patient et ce, dès que possible. Trois experts sur quatre suivent l'efficacité et la tolérance des médicaments et deux sur quatre continuent le suivi au long cours. Le passage de la condition «novice » à la condition « expert » n'est donc pas linéaire.

Dans la dimension 2, "Interaction avec un autre médecin », les novices disent déléguer la prise en charge des patients à un autre professionnel jugé compétent, tandis que les experts adoptent une pratique multiple, composée prioritairement d'échanges et de prise de conseils auprès de collègues. Les 
professionnels expérimentés se différencient des novices par l'apparition de la composante coopération tandis que, d'après les entretiens, la délégation diminue et que les échanges augmentent. L'évolution semble cohérente : l'aspect de délégation observé chez les novices peut traduire un manque d'autonomie ou d'assurance, tandis que les experts sont des professionnels pouvant être sollicités, notamment par des novices. Les médecins de la catégorie expérimentée tendent à agir sans prendre l'avis de leurs collègues ; en revanche, ils délèguent moins et coopèrent plus que les novices.

Dans la dimension 3, «Recherche d'informations », les disparités sont flagrantes : les novices ont une démarche peu variée, uniquement basée sur la lecture - et il s'agit d'une lecture critique, telle qu'elle est enseignée à la faculté. La catégorie expérimentée diversifie sa pratique en intégrant les rencontres avec les visiteurs médicaux et les collègues. L'information est puisée dans la lecture, mais ils sont moins nombreux à dire recourir à l'examen critique des données recueillies que les novices. Chez les experts, l'activité, diversifiée, inclut la nécessité d'exercer son esprit critique.

\section{5.- Structure conceptuelle de la situation}

L'activité de médecins hospitaliers prescripteurs de neuroleptiques concernant le suivi des patients peut, finalement, être représentée sous la forme d'un référentiel de l'activité, fondé sur le discours des experts (Cf. Annexe 4). Ce dernier représenterait la structure conceptuelle de la situation dans l'activité de prescription de neuroleptique dans l'institution étudiée.

\section{4.- Discussion}

Concernant les résultats obtenus, l'action a pu être décrite grâce à trois dimensions issues de l'analyse du contenu des entretiens. Ces trois dimensions regroupent la totalité des savoirs d'action identifiés dans les entretiens.

Dans ce cadre, l'analyse des différentes conceptions de l'activité, notamment la comparaison entre les experts et les autres professionnels, met en lumière trois types de points d'améliorations potentielles concernant la pratique de prescription des neuroleptiques : l'adaptation au contexte local des référentiels médicaux basés sur l'estimation du niveau de preuve scientifique et des avis d'experts ; la mise en œuvre d'une pratique professionnelle fondée sur la communication et l'interdisciplinarité ; le développement de l'analyse critique.

Les recommandations officielles concernant la prescription des neuroleptiques évoquent la surveillance du patient par des bilans mais se limitent à la réalisation du bilan initial, sans aborder sa réactualisation à plus long terme. L'activité des médecins novices puis expérimentés se conforme partiellement à ces recommandations. En revanche, l'activité des experts dépasse le cadre de ces recommandations, en évoquant la réactualisation du bilan au long cours, chez des patients amenés à être hospitalisés de nombreuses fois ou sur de longues périodes. Ce manque de pertinence des prescriptions par rapport au contexte particulier de la pratique de ces professionnels détermine, selon Caniard (2002), le défaut d'adhésion des professionnels aux recommandations médicales. Nos résultats renvoient à la nécessité de l'adaptation des recommandations au contexte de la pratique - ici, la structure hospitalière psychiatrique, qui suit au long cours des patients chroniques - afin de définir un référentiel pertinent et opérant.

Le second point d'amélioration, pour les novices et les professionnels expérimentés, concerne la pratique collective : il s'agit de savoir faire appel à un collègue en cas de décision difficile, de pathologies sous-jacentes moins bien connues, telles que le diabète, l'insuffisance cardiaque ; ceci non pas dans l'unique but de déléguer la prise en charge d'un patient, mais plutôt dans le but d'échanger, de coopérer, comme l'indiquent les experts. Le développement d'un réseau de communications entre professionnels est identifié par Matillon, Le Bœuf et Maisonneuve (2005) comme un passage obligé pour développer l'interdisciplinarité et créer ainsi la possibilité d'un échange de savoirs et d'expé- 
riences qui participe à l'amélioration des pratiques médicales et de la qualité de soins. Nos résultats vont dans ce sens tout en attirant l'attention sur le fait que, sans intégration dans un réseau, des actions réalisées par les novices et les experts semblent disparaître chez des praticiens expérimentés.

Le dernier point d'amélioration concerne l'exercice de l'analyse critique par les médecins. Selon Matillon (2002), ce point est fondamental tant pour l'apprentissage qu'il nécessite que pour les capacités pédagogiques qu'il génère, qui irriguent à leur tour la pratique quotidienne. Nos résultats identifient à ce propos deux éléments constitutifs de l'expertise : la lecture critique de l'abondante littérature médicale et la prise de distance face au discours, toujours empreint de marketing, diffusé par les visiteurs médicaux de l'industrie pharmaceutique. D'où l'intérêt de concevoir des dispositifs de formation fondés sur les débats entre professionnels.

La mise en évidence d'experts et de novices a permis de décrire les professionnels grâce à une combinaison d'indicateurs conçus dans le cadre de cette recherche. Cela permet de faire abstraction des biais institutionnels pouvant intervenir dans leur désignation : âge, ancienneté, grade de chef de service, de praticien hospitalier, de professeur. Ces résultats, extrapolés à l'institution, permettent de définir les professionnels à cibler en priorité lors de la mise en place d'interventions visant à améliorer l'observance des recommandations de bonnes pratiques cliniques : dans cette recherche, les praticiens ayant moins de six mois d'ancienneté dans l'institution, quelle que soit leur ancienneté dans la profession, apparaissent comme un public prioritaire pour ce genre d'actions de formation.

Concernant la méthode, les entretiens basés sur une ordonnance réelle que le professionnel a luimême établie ont l'avantage de confronter chaque professionnel à son activité, et non pas à un cas clinique factice cherchant à reproduire l'activité. L'obtention d'une ordonnance lors d'entretiens réalisés sur le lieu de travail des médecins n'est pas difficile. Ce choix s'est avéré pertinent, permettant d'amorcer puis de réguler l'entretien en le recentrant sur l'activité et le domaine de recherche et en le limitant à la prescription de médicaments neuroleptiques et à leur suivi.

Le respect de l'éthique, annoncé dès la prise de contact avec le professionnel, a permis à chaque médecin prescripteur d'être assuré qu'aucune information nominative évoquée en entretien ne serait divulguée, que les noms de villes et de personnes seraient rendus anonymes lors de la retranscription des entretiens : au final, la problématique du secret professionnel n'a pas constitué un obstacle à cette recherche.

L'analyse de contenu, nécessitant une retranscription des entretiens, se justifiait dans cette recherche exploratoire cherchant à analyser une activité professionnelle. Le nombre élevé de savoirs d'action identifiés a nécessité un travail important de catégorisation ayant abouti au référentiel de l'activité. Le volume de données à traiter constitue toujours un problème avec ce genre de méthodologie qualitative.

Les concepts de la psychologie ergonomique et de la didactique professionnelle - tâche prescrite, tâche effective, activité constructive, schème, compétences, modèle opératif, structure conceptuelle - se sont révélés adaptés à l'analyse de l'activité de médecins prescripteurs. Le référentiel de l'activité découle de l'analyse des modèles opératifs des sujets de l'étude, et notamment de ceux qui sont reconnus comme experts. Bâtis à partir des verbalisations des professionnels interrogés sur leur activité, de tels référentiels représentent une avancée dans une profession où il existe des recommandations générales sur de grands thèmes mais où la prescription concernant la pratique quotidienne reste incomplète.

L'échantillon restreint de l'étude ne permet pas de traitement statistique des données. Les résultats, issus d'un faible effectif de médecins d'une même institution, restent donc limités au contexte de cette institution et ne doivent être extrapolés qu'avec précaution. 


\section{5.- Conclusion et perspectives}

L'amélioration des pratiques médicales relève de trois types de stratégies (François, \& Labarère, 2001) : la standardisation des pratiques, l'approche par problèmes et la formation continue. Concernant la standardisation des pratiques, qui repose sur l'élaboration de normes professionnelles (recommandations de pratique clinique, conférences de consensus, références médicales), la diffusion simple de recommandations n'a que peu d'impact sur les pratiques professionnelles (Durieux, 1998). L'implantation des recommandations passe par une évaluation des pratiques dont la méthode de référence est l'audit clinique, avec retour d'information vers les praticiens. L'investissement à consentir pour une telle stratégie en limite l'utilisation. L'approche par problèmes consiste à identifier et à étudier les erreurs générées par le système pour faire progresser les pratiques et la qualité des soins. Cette approche s'inscrit dans le cadre théorique de l'amélioration continue de la qualité et de la gestion totale de la qualité (Berwick, 1996). La dernière stratégie concerne la formation médicale continue, une nécessité pour accompagner et nourrir les changements de pratiques en santé.

Cette étude propose un cadre conceptuel complémentaire, celui de la didactique professionnelle, comme stratégie d'amélioration des pratiques professionnelles en milieu médical. Ce cadre permet de confronter tâche prescrite et tâche effective et de mettre ainsi en évidence les écarts à la pratique de référence : il ouvre un espace pour une pratique réflexive (Comment je me situe par rapport au standard ?) et pour une confrontation avec la pratique de ses pairs (Comment je me situe par rapport aux autres professionnels de ma communauté ?). Deux types de plus-values en ressortent : l'ouverture d'une communication entre collègues et l'intégration d'un référentiel de pratique « ascendant». En termes de communication, la confrontation régulière des professionnels, experts et autres, lors de séances de réflexion et d'analyse critique d'articles ou d'interventions, de partage d'expériences, amène l'instauration, à plus long terme, de partenariats, voire de réseaux entre ces médecins, centrés sur un même but, la prise en charge optimale du patient. Rejoignant le point de vue de Zarifian (2004) et de Pastré (2005), notre recherche montre que le professionnel ayant organisé son activité au sein d'un réseau est un professionnel expert.

Le cadre de la didactique professionnelle offre une stratégie originale d'intégration, par les professionnels, d'un référentiel de pratique. D'une part, l'écart constaté initialement entre tâche prescrite et tâche effective se comprend mieux lorsqu'il est replacé dans le contexte de l'activité médicale, constituée de situations dynamiques pour lesquelles la prescription de la tâche est nécessairement incomplète. D'autre part, l'accès à cette activité par entretiens, basés sur des ordonnances réelles, permet de recueillir des savoirs d'action exprimés par les professionnels et compense l'impossibilité, pour un chercheur, d'observer ou de simuler ce type d'activité. Leur analyse, incluant la détermination des professionnels experts, aboutit à l'élaboration d'un référentiel de l'activité telle qu'elle est conçue par les praticiens. La pratique des experts semble prendre en compte à la fois la singularité du patient et la systématisation du suivi préconisée par les recommandations. La structure conceptuelle de l'activité, à partir de l'analyse des entretiens des experts, montre que ces derniers « dépassent le patient» afin de prendre en compte de larges aspects de la situation: organisation de l'institution, évolution de la pathologie. La pratique des experts déborde alors les recommandations dans la mesure où ils s'y réfèrent mais les complètent, grâce aux compétences acquises dans le métier. Un référentiel tel que celui auquel aboutit cette étude pourrait donc constituer un outil complémentaire pour la pratique médicale, puisque sont mises au jour non seulement les tâches mais aussi la façon dont elles sont conduites et réalisées. Des étapes critiques dans le développement des compétences apparaissent et peuvent, dès lors, susciter des réflexions, au sein d'une institution donnée, sur les moyens de faciliter leur acquisition, à travers de nouvelles organisations du travail ou de nouveaux modes de formation. 


\section{RÉFÉRENCEMENT}

Grass, A., Grangeat, M., \& Allenet, B. (2007). Analyser l'activité de médecins hospitaliers pour concevoir la formation : le cas de la prescription de neuroleptiques. @ctivités, 4 (1), pp 30-48, http://www.activites. org/v4n1/v4n1.pdf

\section{RÉFÉRENCES}

Anceaux, F., \& Beuscart-Zephir, M.-C. (2002). La consultation préopératoire en anesthésie : gestion de la prise d'informations et rôle des données retenues dans la planification du processus d'anesthésie. Le Travail Humain, 65, 59-88.

Amalberti, R. (2001). La maîtrise des situations dynamiques. Psychologie française, 46(2), $107-$ 118.

Barbier, J.-M., \& Galatanu, O. (Eds.) (2004).Les savoirs d'action : une mise en mots des compétences? Paris: L'Harmattan.

Beaulieu, M.-D., Rivard, M., Hudon, E., Saucier, D., Remondin, M., \& Favreau, R. (2003). Using standardized patients to measure professional performance of physicians. International Journal for Quality in Health Care, 15(3), 251-259.

Bedouch, P., Labarère, J., Chirpaz, E., Allenet, B., Lepape, A., Fourny, M. et al. (2004). Compliance with guidelines on antibiotic prophylaxis in total hip replacement surgery: results of a retrospective study of 416 patients in a teaching hospital. Infection Control and Hospital Epidemiology, 25, 302-307.

Berwick D.M. (1996) A primer on leading the improvement of systems. British Medical Journal, 312, 619-622.

Boreham, N. (2002). Professionalization and work process knowledge in the United Kingdom's National Health Service. In N. Boreham, M. Fisher, \& R. Samurçay (Eds.), Work process knowledge (pp. 171-182). London: Routledge.

Boreham, N., Mawer, G., \& Foster, R. (1996). Medical diagnosis from circumstantial evidence. Le Travail Humain, 59, 69-85.

Boreham, N., Foster, R., \& Mawer, G. (1992). Strategies and knowledge in the control of chronic illness. Le Travail Humain, 55,15-34.

Bourgeois, M.-L. (2003). Les schizophrénies. Paris: PUF.

Caniard, O. (2002). Les recommandations de bonnes pratiques : un outil de dialogue, de responsabilité et de diffusion de l'innovation. Rapport à M. B. Kouchner, Ministre de la Santé, 56-58. $\mathrm{http}: / /$ www.sante.gouv.fr/htm/actu/bon_prat.pdf

Chabannes, J.-P., Benattia, I., Pascal, J.-C., Azorin, J.-M., Vidon, G., Bouhassira, M. et al. (1998). Que recouvre le terme "stabilisation » des patients schizophrènes? L'Encéphale, 24, 331356.

Coulet, J.-C., \& Chauvigné, C. (2005). Passer d'un référentiel de compétences à une ingénierie de formation. Education Permanente, 165, 101-113.

Demange, C., Di Sandro, F., Coince, F., \& Philippe, S. (1999). Observation des pratiques de prescription des héparines de bas poids moléculaire en médecine. Journal de Pharmacie Clinique, 18(2), 156-160.

Durieux P. (1998). Comment améliorer les pratiques médicales ? Approche comparée internationale. Les dossiers de l'Institut d'Études des Politiques de Santé. Paris: Flammarion.

Faure, S., \& Gonzalez, M. (1988). L'acquisition d'informations en situation d'interprétation médicale de résultats d'un examen biologique. Psychologie française, 33(3), 177-186. 
Fédération Française de Psychiatrie. (2003). Selon la méthodologie de l'Agence Nationale d'Accréditation et d'Evaluation en Santé, avec le soutien de la direction de la Direction Générale de la Santé. Conférence de consensus. Schizophrénies débutantes : diagnostic et modalités thérapeutiques.

François, P., \& Labarère, J. (2001). Les processus d'évaluation et d'amélioration des pratiques médicales au Québec. La Presse Médicale, 30, 224-228.

Glikman, J., Pazart, L., Casadebaig, F., Philippe, A., Lachaux, B., Kovess, V. et al. (1999). Étude de l'impact de la conférence de consensus « Stratégies thérapeutiques à long terme dans les psychoses schizophréniques ». L'encéphale, 25, 558-568.

Godfryd, M. (1993). Vocabulaire psychologique et psychiatrique. Paris: PUF.

Grangeat, M. (2006). Formation continue et développement des compétences des enseignants. Education Permanente, 166, 75-88.

Grangeat, M., \& Chakroun, B. (2005). How teachers implement collective activities: on ad-hoc basis or through anticipation? Symposium 'Professional didactic and teaching activity'. Conference proceedings of International conference "What a difference pedagogy makes?", University of Stirling, Scotland, Vol. 2, pp. 720-727.

Grangeat, M., \& Munoz, G. (2006). Le travail collectif des enseignants : activités de coopération et de partenariat d'enseignants de l'éducation prioritaire. Formation Emploi, 95, 94-95.

Gury, C., Ames, D., Camm, J., Cook, P., Falkai, P., Hurley, R. et al. (2003). Comment minimiser les risques associés à un allongement significatif de l'intervalle QTc chez les personnes soufrant de schizophrénie. Recommandations officielles du Cardiac safety in schizophraenia group. Journal de Pharmacie Clinique, 22, 8-17.

Hatchuel, A. (1996, $2^{\circ}$ ed. 2002). Coopération et conception collective. Variété et crises des rapports de prescription. In G. de Terssac, \& E. Friedberg (Eds.), Coopération et conception (pp. 101122). Toulouse: Octarès Éditions.

Haute Autorité de Santé, direction de l'accréditation et de l'évaluation des pratiques. (2005). L'évaluation des pratiques professionnelles dans le cadre del'accréditation des établissements de santé. Disponible sur le site www.has-sante.fr .

Hoc, J.M., Amalberti, R., Cellier J.-M., Grosjean, V. (2004). Adaptation et gestion des risques en situation dynamique. In J.-M. Hoc, \& F. Darses (Eds.), Psychologie ergonomique : tendances actuelles (pp. 15-48). Paris: PUF.

Lachaux, B., Pasi-Delay, P., \& Adouard, D. (1998). La prescription entre art et médecine factuelle. L'Information Psychiatrique, 10, 999-1005.

La revue prescrire. (2006). L’année 2005 du médicament. La Revue Prescrire, 26(269), 147 p.

Leplat, J. (1997). Regards sur l'activité en situation de travail. Contribution à la psychologie ergonomique. Paris: PUF (collection Le Travail Humain).

Marquié, L., Raufaste, E., Mariné, C., \& Ecoiffier, M. (2003). L'erreur de diagnostic en médecine d'urgence : application de l'analyse rationnelle des situations de travail. Le Travail Humain, 66, 347-376.

Matillon, Y. (2002). L'évidence scientifique en médecine. La Presse Médicale, 31, 436-437.

Ménard, S., \& Roy, N. (2004). QT long et neuroleptiques : à propos de 296 cas au centre hospitalier psychiatrique de Saint-Égrève. Thèse de Doctorat en Pharmacie Médecine, UJF, Grenoble.

Matillon, Y., Le Bœuf, D., \& Maisonneuve, H. (2005). Définir et évaluer les compétences des professionnels de santé. Une enquête auprès de 148 organisations. La Presse Médicale, 34, 1703-1709. 
Ministère français des solidarités, de la santé et de la famille (2005). Plan «Psychiatrie et santé mentale 2005-2008 ». Site Internet du ministère français des solidarités, de la santé et de la famille, http://www.sante.gouv.fr/

Pasi-Delay, J., \& Lachaux, B. (1998). Le médicament et ses acteurs : quel(s) partenariat(s) et quelle(s) liberté(s) ? L'Information Psychiatrique, 10, 1021-1026.

Pastré, P. (1999a). La conceptualisation dans l'action : bilan et nouvelles perspectives. Éducation Permanente, 139, 13-37.

Pastré, P. (1999b). Travail et compétences : un point de vue de didacticien. Formation Emploi, 67, 109-124.

Pastré, P. (2004). Le rôle des concepts pragmatiques dans la gestion de situations problèmes : le cas des régleurs en plasturgie. In R. Samurçay, \& P. Pastré (Eds.), Recherches en didactique professionnelle (pp. 17-48). Toulouse: Octarès.

Pastré, P. (2005). La conception de situations didactiques à la lumière de la théorie de la conceptualisation dans l'action. In P. Rabardel, \& P. Pastré (Eds.), Modèles du sujet pour la conception (pp. 73-108). Toulouse: Octarès Éditions.

Prosser, H., \& Walley, T. (2006). New drug prescribing by hospital doctors: the nature and meaning of knowledge. Social Sciences and Medicine, 62, 1565-1578.

Ray-Coquard, I., Chauvin, F., Lurkin, A., Ducimetière, F., Jacquin, J.-P., Agostini, C. et al. (2006). Medical practices and cancer care networks: examples in oncology. Bulletin du Cancer, 93, $13-20$.

Reynaud, M. (1994). L'organisation des soins psychiatriques en France. In M. Reynaud, \& A. Lopez, Évaluation et organisation des soins en psychiatrie (pp. 109-117). Paris: Frison Roche.

Rogalski, J. (1995). Former à la coopération dans la gestion des sinistres. Élaboration collective d'un dispositif d'action. Éducation Permanente, 135, 47-64.

Rogalski, J. (2003). Y a-t-il un pilote dans la classe ? Une analyse de l'activité de l'enseignant comme gestion d'un environnement dynamique ouvert. Recherche en Didactique des Mathématiques, 3(23), 343-388.

Rogalski, J. (2004). La didactique professionnelle : une alternative aux approches de « cognition située » et « cognitiviste » en psychologie des acquisitions. @ctivités, 1 (2), 103-120. http:// www.activites.org/v1n2/html/Rogalski.html

Rogalski, J. (2005). Le travail collaboratif dans la réalisation des tâches collectives. In J. Lautrey, \& J. F. Richard (Eds.), L'intelligence (pp. 147-159). Paris: Hermès.

Sackett, D.L., Rosenberg, W.M., Gray J.A., Haynes, R.B., \& Richardson, W S. (1996). Evidence based medicine: what it is and what it isn't. British Medical Journal, 312, 71-92.

Samurçay, R., \& Hoc, J.M. (1988). Perspectives pour l'aide à l'expertise : De l'analyse du travail à la spécification d'aides à la décision dans des environnements dynamiques. Psychologie française, 33(3), 187-196.

Samurçay, R., \& Rabardel, P. (2004). Modèles pour l'analyse de l'activité et des compétences, propositions. In R. Samurçay, \& P. Pastré (Eds.), Recherches en didactique professionnelle (pp. 163-187). Toulouse: Octarès Éditions.

Sauvagnac, C., Beckendorf V., Lesur, A., Luporsi E., Stines, J., Falzon, P. et al. (1999). Des traitements standards aux cas particuliers : la place des comités de décision thérapeutique en cancérologie. Bulletin du Cancer, 86(9), 767-772.

Spies, T., Mokkink, H., de Vries Robe, P., \& Grol, R. (2004). Which data source in clinical performance assessment? A pilot study comparing self-recording with patient records and observation. International Journal for Quality in Health Care, 16(1), 65-72. 
Staikowski, F., Chouaid, C., Zanker, C., Pevirieri, F., Traineau, I., \& Lagha, S. (1997). Impact de conférences de consensus sur la pratique clinique. Réanimation Urgences, 6(5), 607-612.

Vergnaud, G. (1996). Au fond de l'action, la conceptualisation. In J.-M. Barbier (Ed.), Savoirs théoriques et savoirs d'action (pp. 275-292). Paris: PUF.

Vermersch, P. (2004). Aide à l'explicitation et retour réflexif.Éducation Permanente, 160, 71-80.

Weill-Fassina, A., \& Benchekroun, T.H. (Eds.) (2000). Le travail collectif-Perspectives actuelles en ergonomie. Toulouse: Octarès Éditions.

Zarifian, P. (2004). Le modèle de la compétence. Trajectoire historique, enjeux actuels et propositions. Rueil-Malmaison: Liaisons.

RÉSUMÉ

L'étude vise à comprendre l'écart entre l'activité déclarée par les médecins hospitaliers et la tâche prescrite par les textes officiels. Dans le cadre de la didactique professionnelle, l'étude des conceptualisations est réalisée à partir d'entretiens semi-structurés avec 12 médecins d'un même centre hospitalier, distingués selon leur ancienneté et leur niveau d'expertise. Les résultats permettent de construire un référentiel de l'activité experte et d'esquisser des dispositifs de formation fondés sur les interactions entre novices et experts.

\section{MOTS CLÉS}

didactique professionnelle, compétences, structures conceptuelles des situations, entretiens d'explicitation, schizophrénie, prescription médicale de neuroleptiques.

\section{RESUMEN}

Analizar la actividad de los médicos de hospitales para poder diseñar la capacitación: el caso de la prescripción de neurolépticos. El estudio tiene por objeto comprender la divergencia que se da entre la actividad declarada por los médicos hospitalarios y la tarea prescripta por los textos oficiales. En el marco de la didáctica profesional, el estudio de las conceptualizaciones se realiza a partir de entrevistas semi-estructuradas con 12 médicos de un mismo centro hospitalario, distinguidos según su antigüedad y su nivel de pericia. Los resultados permiten construir un referencial de la actividad experta y esbozar los dispositivos de capacitación, basados en las interacciones entre principiantes y expertos.

Palabras clave

Didáctica profesional, competencias, estructuras conceptuales de las situaciones, entrevistas de explicitación, esquizofrenia, prescripción médica de neurolépticos.

Article reçu le 03 octobre 2006, accepté le 27 janvier 2007 


\section{Annexe 1 : Guide d'entretien à propos de l'activité de prescription de neuroleptiques par des médecins hospitaliers.}

Les neuroleptiques, qui ont révolutionné la prise en charge des patients à leur apparition, sont désormais également connus pour leurs effets indésirables, notamment cardiovasculaires, neurologiques. Des études plus récentes mettent en évidence le risque de diabète induit par ces mêmes médicaments.

J'aimerais que vous me parliez de votre activité de prescription de neuroleptiques sous l'angle du suivi, biologique ou clinique, que vous exercez pour vos patients.

Question 10 reformulée pour les entretiens avec les somaticiens : «Est-ce que vous planifiez un suivi biologique ou clinique en collaboration avec les médecins psychiatres de l'institution?».

\begin{tabular}{|c|c|c|}
\hline Thèmes & Sous-thèmes & Questions \\
\hline $\begin{array}{l}\text { Expérience } \\
\text { professionnelle }\end{array}$ & $\begin{array}{l}\text { Part de l'expérience clinique acquise dans } \\
\text { l'organisation de l'activité }\end{array}$ & $\begin{array}{l}\text { 1. Est-ce que votre expérience vous influence dans la } \\
\text { nature du suivi biologique et/ou clinique que vous } \\
\text { exercez maintenant, pour ce patient? }\end{array}$ \\
\hline \multirow{10}{*}{$\begin{array}{l}\text { Législatif et } \\
\text { réglementaire }\end{array}$} & \multirow{2}{*}{$\begin{array}{l}\text { Formation : } \\
\qquad \begin{aligned} \checkmark & \text { Formation universitaire } \\
\checkmark & \text { Formation médicale continue }\end{aligned}\end{array}$} & $\begin{array}{l}\text { 2. Qu'est-ce qui vous est utile, à partir de votre } \\
\text { formation médicale initiale, dans votre pratique du } \\
\text { suivi biologique? }\end{array}$ \\
\hline & & $\begin{array}{l}\text { 3. Comment transposez-vous dans votre pratique les } \\
\text { informations que vous avez pu recueillir dans le } \\
\text { dernier article / dernier congrès / la dernière } \\
\text { formation? }\end{array}$ \\
\hline & \multirow{3}{*}{ 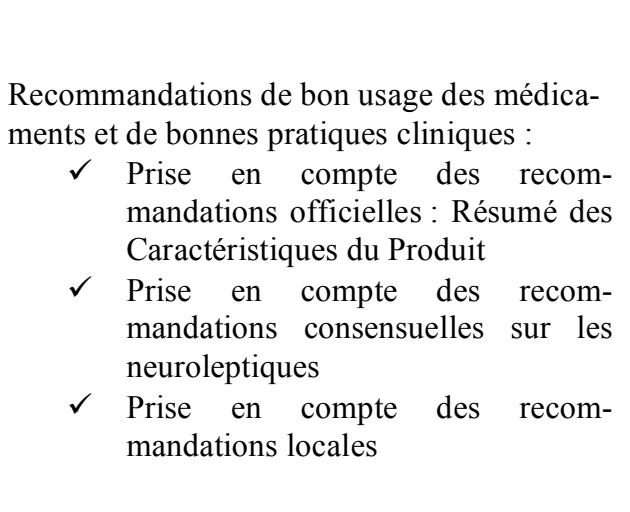 } & $\begin{array}{l}\text { 4. Est-ce que vous ajustez votre démarche aux } \\
\text { recommandations officielles? }\end{array}$ \\
\hline & & $\begin{array}{l}\text { 5. Comment vous conformez-vous aux conférences } \\
\text { de consensus? }\end{array}$ \\
\hline & & $\begin{array}{l}\text { 6. Comment vous conformez-vous aux exigences } \\
\text { locales en matière de suivi du patient? }\end{array}$ \\
\hline & \multirow{2}{*}{$\begin{array}{l}\text { Arguments promotionnels des laboratoires } \\
\text { pharmaceutiques : } \\
\checkmark \quad \text { Analyse critique du discours des } \\
\text { visiteurs médicaux } \\
\checkmark \quad \text { Utilisation de supports publicitaires } \\
\quad \text { des laboratoires pharmaceutiques }\end{array}$} & $\begin{array}{l}\text { 7. Qu'est-ce qui vous est utile dans la visite } \\
\text { médicale pour votre prescription? }\end{array}$ \\
\hline & & $\begin{array}{l}\text { 8. Est-ce que les supports publicitaires remis par les } \\
\text { visiteurs médicaux ont un impact sur votre } \\
\text { pratique? }\end{array}$ \\
\hline & Aspect médico-légal de la profession & $\begin{array}{l}\text { 9. Comment prenez-vous en compte les aspects } \\
\text { médico-légaux de votre profession? }\end{array}$ \\
\hline & $\begin{array}{l}\text { Collaboration avec un médecin somaticien de } \\
\text { l'établissement }\end{array}$ & $\begin{array}{l}\text { 10. Est-ce que vous planifiez un suivi biologique ou } \\
\text { clinique en collaboration avec les médecins } \\
\text { somaticiens de l'institution? }\end{array}$ \\
\hline & $\begin{array}{l}\text { Collaboration avec les autres médecins } \\
\text { somaticiens, extérieurs à l'institution, autour } \\
\text { du suivi du patient }\end{array}$ & $\begin{array}{l}\text { 11. Comment planifiez-vous un suivi biologique ou } \\
\text { clinique en collaboration avec un médecin extérieur } \\
\text { au centre hospitalier, qui peut être un médecin } \\
\text { spécialiste, un médecin généraliste connaissant votre } \\
\text { patient? }\end{array}$ \\
\hline $\begin{array}{l}\text { Conditions } \\
\text { matérielles }\end{array}$ & $\begin{array}{l}\text { Mise à contribution du service de médecine } \\
\text { somatique de l'établissement }\end{array}$ & $\begin{array}{l}\text { 12. Comment tirez-vous parti de l'existence de } \\
\text { médecins somaticiens au sein de l'établis- } \\
\text { sement concernant l'organisation d'un suivi? }\end{array}$ \\
\hline
\end{tabular}




\section{Annexe 2 : Fiche de recueil des caractéristiques du professionnel}

Année d'obtention du diplôme :

Caractéristiques du diplôme :
Ancienneté dans l'institution :

Spécialité médicale :
Ancienneté dans la profession :

Diplômes complémentaires :

sexe $\square \mathrm{F} \square \mathrm{H}$

Age, $\square$ 20-29 a $\square$ 30-39 а $\square$ 40-49 а $\square$ 50-59 a $\square$ 60-69 ans

\begin{tabular}{|l|l|l|l|l|l|}
\hline Type d'activité pratiquée : & $\begin{array}{l}\text { Centre } \\
\text { Hospitalier }\end{array}$ & $\begin{array}{l}\text { Centre } \\
\text { Hospitalier } \\
\text { Universitaire }\end{array}$ & Clinique & Libéral & $\begin{array}{l}\text { Centre Médico } \\
\text { Psychologique }\end{array}$ \\
\hline Auparavant & & & & & \\
\hline Actuellement & & & & \\
\hline
\end{tabular}

Participation à :

\begin{tabular}{|l|l|l|}
\hline Participation à ... & Année & Thèmes le cas échéant \\
\hline $\begin{array}{l}\text { 1-Congrès professionnels, } \\
\text { séminaires, journées de } \\
\text { formation }\end{array}$ & $\square 2004$ & \\
\hline $\begin{array}{l}\text { 2-Comité de lecture, } \\
\text { sélection d'interventions }\end{array}$ & $\square 2005$ & \\
\hline $\begin{array}{l}\text { 3-Membre d'un groupe } \\
\text { de travail d'une société } \\
\text { savante }\end{array}$ & $\square 2005$ & \\
\hline $\begin{array}{l}\text { 4-Abonnement, Temps } \\
\text { réservé pour lecture de } \\
\text { revue professionnelle }\end{array}$ & $\square 2005$ & \\
\hline $\begin{array}{l}\text { 5-Membre d'une } \\
\text { association }\end{array}$ & $\square 2005$ & \\
\hline $\begin{array}{l}\text { 6-Enseignant : institut de } \\
\text { formation aux soins } \\
\text { infirmiers, faculté,... }\end{array}$ & $\square 2004$ & \\
\hline $\begin{array}{l}\text { 7-Étudiant : diplôme } \\
\text { complémentaire }\end{array}$ & $\square 2005$ & \\
\hline $\begin{array}{l}\text { 8-Organisation de } \\
\text { manifestations }\end{array}$ & $\square 2005$ & \\
\hline 2004 & $\square 2005$ & \\
\hline
\end{tabular}




\section{Annexe 3 : Obtention d'un score représentant l'implication dans l'activité professionnelle}

Ancienneté dans la profession : $\quad$ Non diplômés 0 point (Internes en médecine)

Diplômés 1 point

Ancienneté dans l'institution : $\quad$ Professionnel arrivé depuis moins d'un an 0 point Professionnel arrivé depuis plus d'un an 1 point

Caractéristiques du diplôme : $\quad$ Pas de diplôme complémentaire $\quad 0$ point

1 à 2 diplômes complémentaires 1 point

De 3 à 5 diplômes complémentaires 2 points

Plus de 5 diplômes complémentaires 3 points

Type d'activité pratiquée : expérience professionnelle (passée ou actuelle) :

Dans 1 à 2 établissements différents 1 point

Dans 3 établissements différents 2 points

Dans plus de 3 établissements différents 3 points

En 2004 et 2005, participation à :

1- Congrès professionnels, séminaires, journées de formation :

Aucun : 0 point Moins d'un par mois : 1 point Plus d'un par mois : 2 points

2- Comité de lecture, de sélection d'interventions : Non : 0 point Oui : 1 point

3- Membre d'un groupe de travail (société savante) : Non : 0 point $\quad$ Oui : 1 point

4- Abonnement, temps réservé pour lecture de revues professionnelles :

Non : 0 point 1 revue : 1 point 2 revues : 2 points

5- Membre d'une association ayant trait à l'activité professionnelle :

Non : 0 point

Oui : 1 point

6- Enseignant : institut de formation aux soins infirmiers, faculté, visiteurs médicaux

Non : 0 point Occasionnellement : 1 point Régulièrement : 2 points

7-Étudiant (diplôme complémentaire) : Non : 0 point Oui : 1 point

8-Organisation de manifestations : Non 0 point

Dans le cadre de l'institution : 1 point

Dans un cadre extérieur à l'institution : 2 points

Score : / 20 
Annexe 4 : Référentiel de l'activité de prescription de neuroleptiques par des médecins hospitaliers

\begin{tabular}{|c|c|c|}
\hline Dimensions & Actions & Règles d'action \\
\hline \multirow{8}{*}{$\begin{array}{l}\text { 1. Suivi biolo- } \\
\text { gique ou clinique } \\
\text { du patient }\end{array}$} & \multirow{2}{*}{ 1.1 Faire un bilan dès que possible } & Faire les examens à l'entrée \\
\hline & & Faire les examens avant traitement \\
\hline & \multirow{2}{*}{$\begin{array}{l}1.2 \text { Adapter la nature et/ou la } \\
\text { fréquence du suivi aux signes } \\
\text { biologiques et/ou cliniques }\end{array}$} & Éviter des examens inutiles \\
\hline & & Être plus attentif aux patients à risque \\
\hline & \multirow[t]{2}{*}{ 1.3 Continuer le suivi au long cours } & $\begin{array}{l}\text { Faire un contrôle annuel en l'absence de } \\
\text { problème }\end{array}$ \\
\hline & & Évaluer l'opportunité d'un contrôle \\
\hline & \multirow{2}{*}{$\begin{array}{l}1.4 \text { Suivre l'efficacité et/ou la } \\
\text { tolérance du traitement }\end{array}$} & Évaluer l'efficacité \\
\hline & & Évaluer la tolérance \\
\hline \multirow{10}{*}{$\begin{array}{l}\text { 2. Interaction } \\
\text { avec d'autres } \\
\text { médecins }\end{array}$} & \multirow{2}{*}{$\begin{array}{l}\text { 2.1 Échanger des informations sur le } \\
\text { patient }\end{array}$} & Connaître le dossier du patient \\
\hline & & Dire des choses importantes \\
\hline & \multirow{2}{*}{$\begin{array}{l}2.2 \text { Prendre conseil auprès des } \\
\text { collègues }\end{array}$} & Orienter des examens complémentaires \\
\hline & & Interpréter des résultats \\
\hline & \multirow{5}{*}{$\begin{array}{l}2.3 \text { Coopérer pour la prise en charge } \\
\text { du patient }\end{array}$} & Récupérer un dossier, des examens antérieurs \\
\hline & & Faciliter la continuité des soins \\
\hline & & $\begin{array}{l}\text { Parler des interférences entre le traitement et un } \\
\text { problème somatique }\end{array}$ \\
\hline & & $\begin{array}{l}\text { Associer le somaticien référent à la prise en } \\
\text { charge psychiatrique }\end{array}$ \\
\hline & & Introduire un nouveau traitement \\
\hline & 2.4 Déléguer la prise en charge & $\begin{array}{l}\text { Ne pas se contenter de déléguer la prise en } \\
\text { charge somatique du patient }\end{array}$ \\
\hline \multirow{14}{*}{$\begin{array}{l}\text { 3. Recherche } \\
\text { d'informations }\end{array}$} & \multirow{5}{*}{ 3.1 Rencontrer des collègues } & Assister à des séances de formation \\
\hline & & Aller dans des congrès \\
\hline & & Interroger des collègues sur leur expérience \\
\hline & & $\begin{array}{l}\text { Participer en équipe à des séances d'information } \\
\text { du patient menées par un laboratoire } \\
\text { pharmaceutique }\end{array}$ \\
\hline & & Recevoir de futurs médecins généralistes \\
\hline & \multirow{4}{*}{ 3.2 Lire } & Lire des cours \\
\hline & & Lire des livres \\
\hline & & Lire des revues médicales \\
\hline & & Lire le dictionnaire VIDAL $®$ \\
\hline & \multirow[b]{2}{*}{ 3.3 Acquérir un esprit critique } & Développer sa lecture critique \\
\hline & & $\begin{array}{l}\text { Rester critique vis-à-vis de l'information des } \\
\text { visiteurs médicaux }\end{array}$ \\
\hline & \multirow{3}{*}{ 3.4 Avoir différentes sources } & $\begin{array}{l}\mathrm{Ne} \text { pas se contenter d'utiliser les supports } \\
\text { publicitaires des laboratoires }\end{array}$ \\
\hline & & Utiliser tous les canaux d'information \\
\hline & & Recevoir des visiteurs médicaux \\
\hline
\end{tabular}

